\title{
Discovering Hidden Blue Ocean Strategy with KeyGraph Machine
}

\author{
Fang-Cheng Hsu ${ }^{1}$, Han-Yuan Lee ${ }^{2}$ and Tzong-Heng Chi ${ }^{1}$ \\ ${ }^{1}$ Department of Information Management, Aletheia University \\ ${ }^{2}$ Graduate School of Management Sciences, Aletheia University
}

\begin{abstract}
Researchers usually focused on using unstructured documents, such as news, scientific literatures, World Wide Web pages, business reports, as input documents of KeyGraph. But the unstructured documents might results in unclear keywords and meaningless keywords. Although traditional document preprocessing could dismiss some of the problems, generating complex and unreadable KeyGraph diagrams were not avoidance. In this research, we applied the concept of chance discovery and KeyGraph to discover hidden blue ocean strategy (BOS) for decision makers who not necessary familiar with the concerned domains. A preprocessing strategy, including develop an operational framework of BOS and design a sentence structure for representing BOS, was proposed to assure the necessary keywords be included in the required documents. Seventy-two documents concerning blue ocean firms were collected as sample cases for developing the operational framework. At last, three experiences were designed for confirming the performance of the proposed preprocessing strategy. Experimental results shown that the subjects were not consistently recognized BOS with traditional approach (used unstructured documents as input documents of KeyGraph). In contrast, the subjects could easily find explicit scenarios of BOS, and recognize a few of implicit scenarios appeared on KeyGraph diagrams with the proposed strategy.
\end{abstract}

Keywords: Chance discovery, Blue ocean strategy, Strategy discovery

\section{Introduction}

The KeyGraph was a tool for automated indexing [5]. However, it has well known for its fruitful works on chance discovery. Ohsawa and McBurney [4] defined "chance is an event or a situation which can effectively contribute to user's decision making." KeyGraph has shown his powerful capacity on drawing scenarios, and visualizing the relations of events (or states, or components) in the target environment [2].

KeyGraph has been used to discover future chances for years; however, researchers usually used unstructured documents, such as, news, scientific literatures, World Wide Web pages and business reports, as inputs of KeyGraph. The unstructured documents always results in unclear keywords and meaningless keywords. Although document preprocessing could dismiss some of the problems, generating complex and unreadable KeyGraph diagrams were not avoidance.

In this research, we applied the concept of chance discovery and KeyGraph to discover hidden blue ocean strategy (BOS) for decision makers who not necessary familiar with the concerned domains. A primary difficulty will arise when applying KeyGraph to discover the hidden BOS: How BOS should be represented and what keywords should be included in required documents for KeyGraph? A coding preprocessing, including developed an operational framework of BOS and designed a sentence structure for representing BOS, was proposed to assure the necessary keywords be included in the required documents.

Seventy two blue ocean firms, which have detailed but unstructured descriptions in two well known books [1, 3], were chosen as sample cases for developing the operational framework of BOS and experimental analysis. Every sample case will be coded into a sentence. All the coded sentences would were grouped and combined into different documents for generating a series of KeyGraph diagrams. At last, three experiments were designed for confirming the performance of the proposed preprocessing strategy.

\section{A KeyGraph Machine for Discovering Hidden BOS}

Blue ocean strategy (BOS), proposed by Kim and Mauborgne [3], is a way to make the competition irrelevant by creating a leap in value for both the firms and its customers. Value innovation, the cornerstone 
of BOS or the key element of BOS, can help firms make giant leaps in the value provided to customers.

BOS have applied across all types of industries from the typical suspects of consumer product goods to $\mathrm{B} 2 \mathrm{~B}$, pharmaceutical, financial services, entertainment, IS/IT, and even defense. It is very interesting to know: 1) were there any differences when applied BOS to different firms? 2) Can we discover any hidden blue ocean strategy among the successful blue ocean firms?

For knowing weather the differences were exist or not and what were the differences (if the differences exist), One of the best way is to provide users a KeyGraph machine so that they can just input documents concerning blue ocean firms and then get some readable BOS scenario diagrams and some hidden BOS scenario diagrams. Because original documents, describing BOS of firms, usually have unclear or meaningless keywords, the problem of how to represent BOS and how to assure required keywords were included in documents for KeyGraph were arisen.

It is believe that a clear structured document is helpful for users discovering some of hidden BOSs with KeyGraph machine. Because the conceptual framework of BOS (Fig. 1) has a well designed structure, it is interesting to design a sentence structure, which can be used to form the original descriptions of the BOSs into structured sentences for KeyGraph, by following the conceptual framework of BOS.

The conceptual framework of BOS is good for both new and existing firms in pursuit of value innovation, but each blue ocean firm in different environment should has its unique way of manipulating the six principles and the four actions. It means that different firms might have its unique keywords describing its principles and actions. We proposed a three-step way of developing the sentence structure as following:

Step 1: Creating a operational framework of BOS

We first collected 72 blue ocean firms which have detailed but unstructured descriptions in two well known books [1, $3]$, and then constructed 25 sub-principles according to actually works of the 72 real cases. The sub-principles composed of the operational framework of BOS (Fig. 2).

Step 2: Coding the operational framework of BOS

Giving all elements of the operational framework a unique code (Fig. 2)

Step 3: Define the sentence structure S
Sentence structure, $\mathrm{S}$, will contain several kinds of codes which representing "what actions the firms have taken and "what principles or sub-principles the firm have followed".

$\mathrm{S}$ is defined as:

$$
\begin{aligned}
& \mathrm{V}=\{\mathrm{V}\}\} \\
& R=\left\{\begin{array}{l}
R 1, R 11, R 12, ., R 16 R 2, R 21, \\
R 22, ., R 24, ., \text { R6,R61,R62, . , R64 }
\end{array}\right\} \\
& \mathrm{A}=\{\mathrm{A} 1, \mathrm{~A} 2, \mathrm{~A} 3, \mathrm{~A}\} \\
& \{S \mid S=V s \cup R s \cup A s, V s \in V, R s \in R, A s \in A\}
\end{aligned}
$$

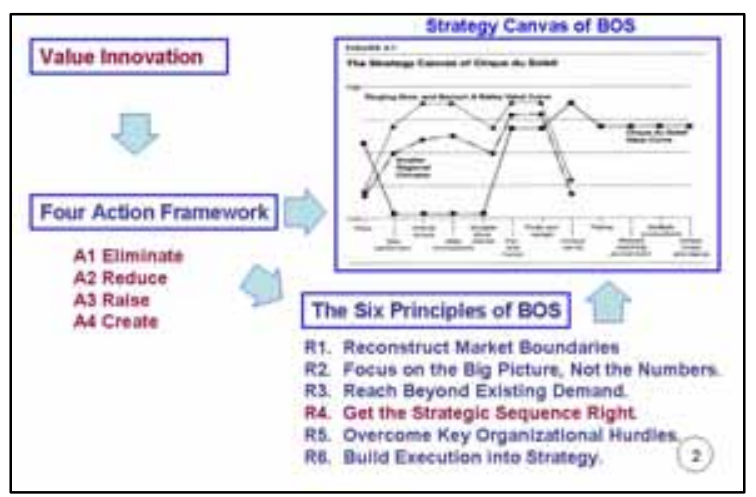

Fig. 1 The Conceptual Framework of BOS

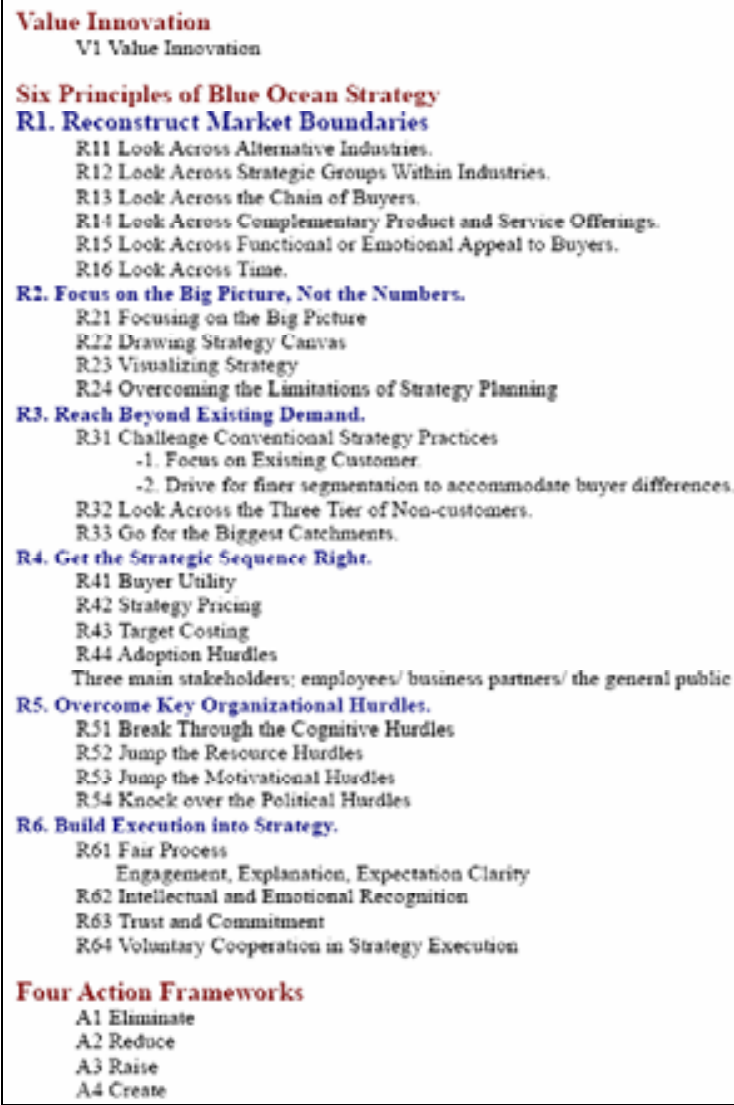

Fig. 2 The Operational Framework of BOS 
To fulfill the three-step way 3 to 5 facilitators were asked to carefully identify each unstructured document what actions the blue ocean firm taken and what principles or sub-principles the blue ocean firm followed; and then coded it into a sentence. For example, If firm A is a blue ocean firm, it took actions A1, A3; and followed the conceptual principle R11, R13, R21, R32, R41, R51, and R52 then the BOS of the firm will be coded as a structured sentence: $\{\mathrm{A} 1$, A3, R11, R13, R21, R32, R41, R51, R52\}. Every firm has it's structured sentence; the sentences will be grouped into documents for discovering hidden chances by KeyGraph..

\section{Case Studies of BOS}

To verify the performance of the proposed preprocessing strategy 72 documents (57 for Worlds and 15 for Taiwan) concerning blue ocean firms in two well known books [1, 3] were collected as sample cases for experimental analysis. The experimental settings of the traditional KeyGraph preprocessing strategies and the proposed strategy are listed in Table 1. Three experiments were designed for confirm the performance of the proposed strategy.

Experiment \#1: whether the readabilities of KeyGraph diagrams generated by the proposed is better than the traditional preprocessing strategies.

Experiment \#2: whether the proposed performed better than the traditional preprocessing strategies in capabilities of recognizing BOS.

Experiment \#3: whether the proposed performed better than the traditional preprocessing strategies in capabilities of recognizing hidden BOS.

Table 1 Experimental settings

\begin{tabular}{|c|c|c|}
\hline \multicolumn{3}{|c|}{ The proposed preprocessing strategy } \\
\hline Documents: & $\begin{array}{l}\text { Sample set \#1 } \\
\text { Sample set \#2 } \\
\text { Sample set \#3 } \\
\text { Sample set \#4 }\end{array}$ & $\begin{array}{l}32 \text { Manufacturing cases in [3] } \\
34 \text { North American cases in [3] } \\
\text { All cases (57 cases) in [3] } \\
15 \text { Taiwan cases in [1] }\end{array}$ \\
\hline $\begin{array}{l}\text { Document } \\
\text { preprocessing: }\end{array}$ & \multicolumn{2}{|c|}{$\begin{array}{l}\text { The proposed coding preprocessing strategy. } \\
\text { Three decision makers with } 5 \text { more year } \\
\text { experiences in strategic planning and one } \\
\text { technician with at least } 1 \text { year experience in } \\
\text { KeyGraph. }\end{array}$} \\
\hline \multicolumn{3}{|c|}{ The traditional strategies } \\
\hline $\begin{array}{l}\text { Documents: } \\
\text { Document } \\
\text { preprocessing: } \\
\text { Subjects }\end{array}$ & $\begin{array}{l}\text { Document com } \\
\text { Same with the }\end{array}$ & $\begin{array}{l}\text { ction and phrase construction. } \\
\text { posed strategy. }\end{array}$ \\
\hline
\end{tabular}

\section{Results of Case Studies}

We grouped the 72 sample cases into 4 categories: 32 cases of manufacturing were categorized as sample set
\#1; 34 North America were categorized as the Sample set \#2; Sample set \#3 was the all 57 World cases, and sample set \#4 was the all 15 Taiwan cases. Every sample set was coded into structured sentences; the coded sentences of sample sets were combined into documents and were used to generate a series of KeyGraph diagrams.

The aim of experiment \#1 was designed to test the readabilities of the generating diagrams. With the proposed preprocessing the 3 subjects had a consistency scenario, spent shorter time to read the KeyGraph, and readability score was higher than the traditional preprocessing.

The results of the experiment \#2 shown that no mater by sample set \#1, sample set \#2 or sample set \#3, the proposed preprocessing strategy was helpful for recognizing the identical explicit scenario of BOS which composed of V1: value innovation, the four action framework (A1, A2, A3, A4), and R41: customer utility, R42: strategic pricing, and R43: target cost (Fig $3 \sim$ Fig 5). It is very interesting to find that R42: strategic pricing was a chase rather than the core principle of BOS in Taiwan cases (Fig 6).

For experiment 3, two scenarios of hidden BOS were created: (1) GROUP \#1(R11: look across alternative industry, R31: challenge the conventional strategy practices,), CHANCE \#1(R44: address adoption hurdles), and GROUP \#2(V1, A1, A2, A3, A4, R41, R42, R43) (Fig. 5), (2) GROUP \#3(R14: look across complementary product and service offerings), CHANCE \#2(R31: to challenge the conventional strategy practices) and GROUP \#4(V1, A1, A2, A3, A4, R32, R42, R12) (Fig. 7)

It is important to mention that after recognizing the 2 hidden BOS the subjects can provide real world cases to verify the two hidden BOS.

Table 2 Results of the experiment 2-1

\begin{tabular}{|c|c|c|c|c|}
\hline \multirow{3}{*}{ Sample set \# } & \multicolumn{4}{|c|}{$\begin{array}{l}\text { Average time spent in Min and Average } \\
\text { readability score in } 100 \text { points scale }\end{array}$} \\
\hline & \multicolumn{2}{|c|}{ The proposed } & \multicolumn{2}{|c|}{ The traditional } \\
\hline & Time & Score & Time & Score \\
\hline$\# 1$ & 17.0 & 87 & 22.7 & 68 \\
\hline \#2 & 19.6 & 85 & 29.0 & 75 \\
\hline \#3 & 14.0 & 88 & 22.3 & 65 \\
\hline$\# 4$ & 13.3 & 85 & 24.0 & 74 \\
\hline \multirow{2}{*}{ Sample set \# } & \multicolumn{4}{|c|}{ Has a consistence scenario? } \\
\hline & \multicolumn{3}{|c|}{ The proposed } & \\
\hline$\# 1$ & \multicolumn{3}{|c|}{ Yes } & No \\
\hline \#2 & \multicolumn{3}{|c|}{ Yes } & No \\
\hline \#3 & \multicolumn{3}{|c|}{ Yes } & No \\
\hline \#4 & \multicolumn{2}{|c|}{ Yes } & \multicolumn{2}{|c|}{ No } \\
\hline
\end{tabular}

Table 3 Results of the experiment 2-2

\begin{tabular}{|c|c|c|}
\hline \multirow{2}{*}{ Sample set \# } & \multicolumn{2}{|c|}{ What BOS has consistently recognized? } \\
\cline { 2 - 3 } & The proposed & The traditional \\
\hline$\# 1$ & (V1,A1,A2,A3,A4,R41,R42,R43) & NA \\
$\# 2$ & (V1,A1,A2,A3,A4,R41,R42,R43) & NA \\
$\# 3$ & (V1,A1,A2,A3,A4,R41,R42,R43) & NA \\
$\# 4$ & (V1,A1,A2,A3,A4,R41,R43) & NA \\
\hline
\end{tabular}




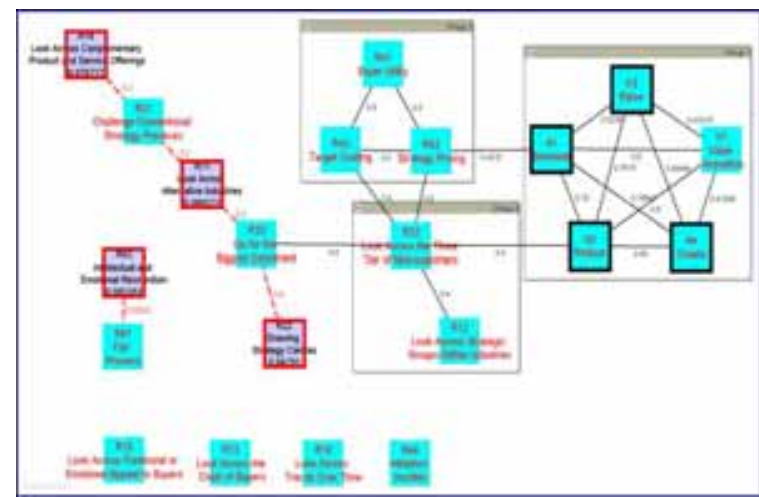

Fig.3 Sample \#1 with the proposed strategy

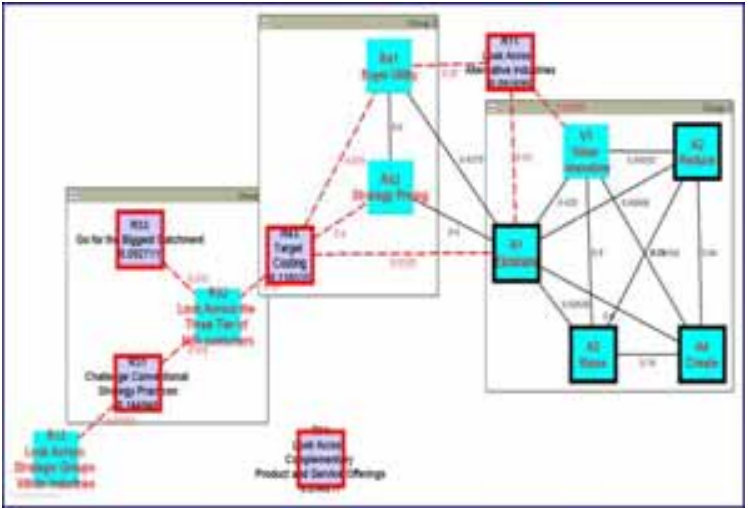

Fig. 4 Sample \#2 with the proposed strategy



Fig.5 Sample \#3 with the proposed strategy

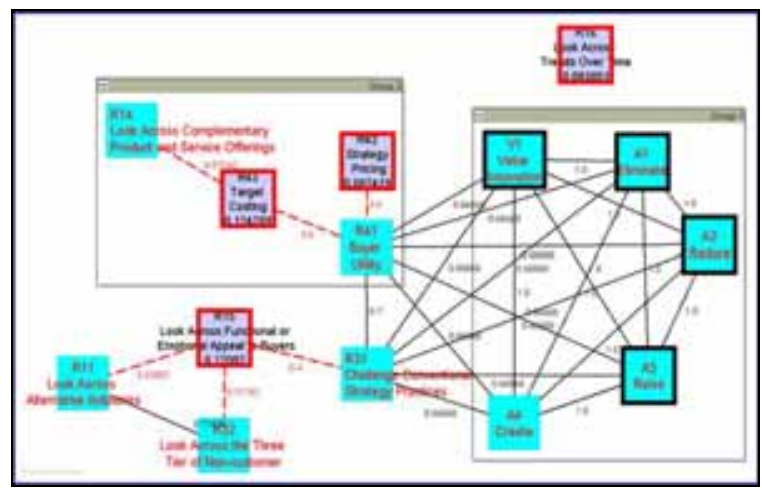

Fig.6 Sample \#4 with the proposed strategy

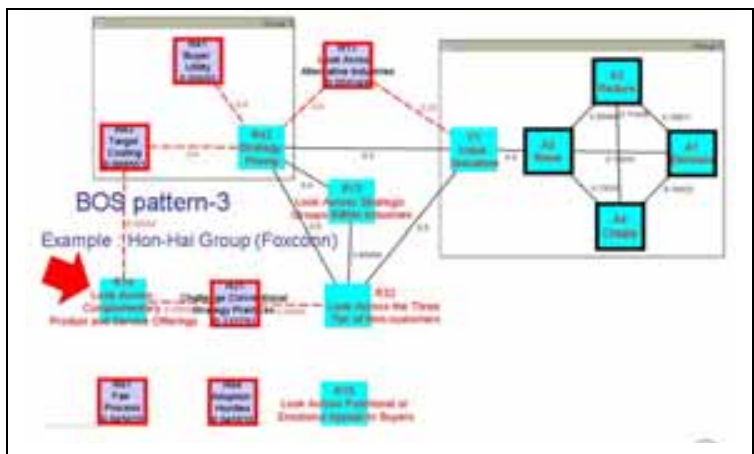

Fig. 7 Hidden BOS with the proposed strategy

\section{Conclusions}

The objective of this research is to provide decision makers a KeyGraph machine for discovering hidden BOS. If the generated diagrams of the machine full of meaningless or unclear keywords, the decision makers would suffer from discovering hidden BOS on poor readable diagrams. We proposed a preprocessing strategy for assuring the necessary keywords be included in the required documents. The initial results shown that the subjects could easily find not only explicit scenarios of BOS, but also recognize a few of implicit or hidden scenarios of BOS appeared on KeyGraph diagrams generated with the proposed strategy; In contrast, they were not consistently recognized the explicit BOS on diagrams generated with the traditional $g$ strategies. The results show that the proposed strategy is a workable way to improve the readabilities of KeyGraph diagrams.

\section{References}

[1] P. Y. Chu, Blue Ocean Strategy in Taiwan, the World Publishing, 2006.

[2] R. Fruchter, Y. Ohsawa, and N. Matsumura, "Knowledge reuse through chance discovery from an enterprise design-build enterprise data store,” New Mathematics and Natural Computation 1(3), 2005, pp. 393-406.

[3] W. C. Kim, and R. Mauborgne, Blue Ocean Strategy-How to Create Uncontested Market Space and Make the Competition Irrelevant, Harvard Business School Press, 2005.

[4] Y. Ohsawa, "Modeling the Process of Chance Discovery," in Ohsawa and McBurney (Eds.) Chance Discovery, Springer, 2003, PP. 2-15.

[5] Y. Ohsawa, N. E. Benson and M. Yachida, "KeyGraph: Automatic Indexing by Cooccurrence Graph based on Building Construction Metaphor," Proceedings of the Advanced Digital Library Conference (IEEE ADL'98), 1998, pp.12-18. 